\title{
¿Existe relación entre el tamaño del grupo de investigación y su rendimiento científico? Estudio de caso de una universidad española
}

\author{
Álvaro Cabezas-Clavijo*, Evaristo Jiménez-Contreras*, Emilio Delgado López-Cózar* \\ *EC3: Evaluación de la Ciencia y de la Comunicación Científica, Departamento de Información y Comunicación, Universidad de Granada. \\ Correo-e: acabezasclavijo@gmail.com; evaristo@ugr.es; edelgado@ugr.es
}

Recibido: 17-05-2012; 2a version: 10-09-2012; Aceptado: 4-2-2013.

Cómo citar este artículo/ Citation: Cabezas-Clavijo, A.; Jiménez-Contreras, E.; Delgado López-Cózar, E. (2013). ¿Existe relación entre el tamaño del grupo de investigación y su rendimiento científico? Estudio de caso de una universidad española. Revista Española de Documentación Científica, 36(2):e006. doi: http://dx.doi.org/10.3989/redc.2013.2.984

Resumen: Se presenta un análisis del rendimiento de grupos de investigación desde una perspectiva bibliométrica. Tomando como caso de estudio los grupos de investigación de la Universidad de Murcia, salvo los del área de Humanidades $(n=258)$, se indaga en la relación entre el tamaño del grupo de investigación, y su rendimiento científico, usando para ello medidas bibliométricas absolutas y relativas. Los resultados indican que los grupos con más miembros muestran valores absolutos de producción, visibilidad e impacto más elevados que el resto de grupos, como consecuencia de su mayor tamaño. En lo que respecta a los indicadores relativos, se concluye que existe relación estadísticamente significativa entre tamaño del grupo y porcentaje de publicación en revistas del primer cuartil, así como con el valor de citas normalizado. Estos resultados vienen dados por el área de Ciencias Sociales donde la productividad per cápita también se ve afectada positivamente por el mayor tamaño del grupo de investigación. En las restantes áreas, sin embargo, no hay evidencia estadística de que los indicadores relativos estén influidos por el tamaño del grupo de investigación. Finalmente, se discuten las implicaciones de estos resultados para la toma de decisiones en política científica.

Palabras clave: Productividad; impacto; visibilidad; actividad; tamaño; grupos de investigación; análisis bibliométricos; evaluación de la investigación; universidad; España.

\section{Is there a relation between size and scientific performance of research groups? A Spanish university as a case study}

\begin{abstract}
An analysis of the performance of research groups from a bibliometric point of view is presented. We take into account the 258 research groups from the University of Murcia as a case study (excluding those from Humanities), in order to delve into the relationship between the size and the scientific performance of research groups, using absolute and relative bibliometric measures. Results point out that groups with more members show higher absolute values of production, visibility and impact as a consequence of their bigger size. Regarding relative indicators, we conclude that there is a statistically significant relationship between group size and the share of first quartile publications, as well as with the normalised citation impact. These results are determined by the Social Sciences area, where also per capita output is positively affected by group size. However, no statistical evidence has been found in the other areas regarding the possible influence of group size upon relative indicators. Finally, implications of these results for decision-making in research policy are discussed.
\end{abstract}

Keywords: Productivity; impact; visibility; activity ;size; research groups; bibliometric analysis; research evaluation; university; Spain.

Copyright: () 2013 CSIC. Este es un artículo de acceso abierto distribuido bajo los términos de la licencia Creative Commons Attribution-Non Commercial (by-nc) Spain 3.0. 


\section{INTRODUCCIÓN}

Una de las características distintivas de la ciencia desde el siglo XX es el salto desde la labor individual de los científicos al trabajo en colaboración. Aunque no de forma exclusiva, dicho quehacer se ha articulado principalmente a través del grupo de investigación. Este nivel de análisis ha sido, sin embargo, frecuentemente ignorado en los análisis bibliométricos, que han fijado más el foco en el rendimiento de unidades como departamentos o universidades, probablemente por la dificultad de contar con censos de grupos de investigación y la imposibilidad de recuperar este dato a partir de los campos de afiliación de las bases de datos internacionales, ya que los investigadores no suelen consignarlo. No hay que olvidar, sin embargo, que un investigador pasa una parte importante del tiempo trabajando con sus colaboradores más inmediatos dentro del grupo de investigación. Lee y Bozeman (2005) sobre una muestra de académicos norteamericanos cifran en un $51 \%$ el tiempo invertido en investigación con los miembros del propio grupo. Esta importancia del grupo de investigación también se percibe hoy día a través de su presencia web, ya que, en el caso de las universidades españolas, casi la tercera parte de los URLS pertenecen a los grupos de investigación (OrduñaMalea, 2011). Este trabajo, pues, se centra en el grupo de investigación al tratarse de la principal unidad ejecutora de la investigación en los centros de $I+D$, y particularmente en las universidades (von Tunzelmann y otros, 2003).

Pese a la dificultad en encontrar una definición de consenso para grupo de investigación (Rey-Rocha y otros, 2008), podemos seguir a estos autores cuando afirman que "un grupo de investigación es una asociación estable, aunque dinámica, en torno a una línea de investigación, de personal de investigación generalmente de una misma institución, que comparte el mismo sentido de pertenencia y mantiene unas relaciones basadas en las interacciones y la colaboración permanente". Esta definición pone de manifiesto que un grupo de investigación no lo forman únicamente los investigadores seniores del grupo, sino también el personal en formación, técnicos o colaboradores del mismo. Sin embargo, desde el punto de vista de la medición bibliométrica es complejo determinar el rol que desempeñan estas figuras, por lo que es frecuente estudiar el rendimiento únicamente de los miembros de mayor rango académico del grupo (von Tunzelmann y otros, 2003).

La definición citada señala también la importancia de los aspectos sociales en la configuración de un grupo de investigación, que se conforman libremente y de mutuo acuerdo entre los propios investigadores. La pertenencia a un grupo de investigación se identifica con una serie de factores tanto sociales como de índole científica, y que pueden redundar en el rendimiento del mismo. Así, uno de los factores que se relacionan con el grupo de investigación es su grado de cohesión interna. El sentido de pertenencia, la identidad, el auto reconocimiento y un cierto sentimiento de fidelidad son algunas de las características que permiten identificar a los grupos cohesionados (Rey-Rocha y otros, 2008). En este sentido, autores como Etzkowitz (1992) califican de cuasi-familiar la relación que se produce en los laboratorios de muchos grupos de investigación, dada la cantidad de tiempo que se trabaja y las relaciones que surgen entre los miembros. La intensidad emocional, la confianza mutua y la reciprocidad en las relaciones son otros de los factores personales que deben existir para que dos investigadores establezcan un vínculo fuerte entre ellos (Granovetter, 1973), si bien no todas las relaciones que se establecen entre los miembros de un grupo de investigación son de esta naturaleza.

En la literatura se han descrito múltiples factores que pueden afectar el rendimiento del grupo de investigación, siendo la relación entre el tamaño de los grupos y la productividad uno de los aspectos más estudiados. En su revisión, Cohen (1991) no encuentra que haya un tamaño óptimo para un grupo de investigación, ya que detecta como la producción total de los grupos crece de manera lineal respecto al tamaño de éstos, manteniéndose inmutable la producción per cápita. Señala asimismo que uno de los elementos clave es cómo medir la productividad. Usando la coautoría como elemento discriminador de los grupos, Seglen y Aksnes (2000) llegaron a la misma conclusión en su estudio de la microbiología noruega. Aunque con el aumento del tamaño de grupo, aumentaba su output global, la productividad per cápita de los miembros se mantenía constante. En el informe técnico de la SPRU (Science and Technology Policy Research) británica (von Tunzelmann y otros, 2003) sin embargo se encuentran evidencias de que para grupos de investigación pequeños, la productividad llega a su máximo cuando se alcanzan las cinco personas, manteniéndose una ratio constante a partir de dicho tamaño. Johnston (1994) por su parte afirma que para competir a nivel internacional el tamaño de grupo mínimo, entendido este como el de los científicos de mayor grado científico, estaría entre cuatro y seis, que deberían acompañarse de aproximadamente otras cuatro personas entre personal técnico y becarios. Así, pues existen evidencias contradictorias acerca del efecto del tamaño del grupo de investigación en el rendimiento investigador. El estudio de la SPRU advierte de otros factores influyentes en la productividad de los grupos, como las diferencias de tamaño que pueden producirse entre disciplinas, la edad de los investigadores o la financiación que consiguen los grupos de investigación. En el estudio de Dundar y Lewis (1998) destinado a medir los factores que afectan a la productividad científica en los departamentos de las instituciones de educación superior, se asevera que el tamaño del departamento es un factor crítico en cuanto a la investigación ya que el mayor tamaño de éste se 
relaciona con una mayor productividad per cápita. Esta circunstancia redundaría en un mayor poder interno en la institución, lo que se podría traducir en más y mejores recursos para la investigación; en este caso el tamaño seria un método indirecto para la consecución de recursos humanos, económicos y materiales, para la investigación, además sería un factor de atracción de potenciales colaboradores, lo que conduciría a una mayor productividad de estos departamentos. Otros factores identificados en dicho trabajo como influyentes en la productividad son el disponer de un alto porcentaje de profesores catedráticos así como contar con una ratio elevada de profesores en el departamento dedicado a tareas investigadoras.

Respecto a la relación entre tamaño de grupo y visibilidad e impacto de la investigación, es llamativo que esta relación se ha estudiado mucho menos que la existente entre tamaño y productividad. Así, algunos trabajos publicados se han centrado en estudiar la ventaja acumulativa en los grupos de investigación, hallando que son los grupos menos productivos los que parecen verse más beneficiados por el aumento del tamaño, si bien en estos trabajos el tamaño se mide por la producción total y no por el número de miembros que componen el grupo (van Raan, 2006). Por su parte, desde un punto de vista matemático (Kenna y Berche, 2011; Kenna y Berche, 2012) se ha hallado que existe un tamaño óptimo para los grupos de investigación, y que éste presenta importantes oscilaciones en función de la disciplina. Estos autores afirman que la calidad de los grupos aumenta con su tamaño, hasta alcanzar un determinado umbral en el que los grupos son tan grandes que se subdividen actuando en la práctica como si fueran varios. Hay que señalar que en estos análisis no se emplea el concepto de calidad o impacto desde un punto de vista bibliométrico sino desde la visión del análisis de expertos.

En clave nacional, no son muchas las evaluaciones bibliométricas centradas en grupos de investigación. Cabe citar los trabajos de Rey-Rocha que miden la relación entre la consolidación de los grupos de investigación y su productividad para Biología y Biomedicina (Rey-Rocha y otros, 2006) y más recientemente para Química (Rey-Rocha y otros, 2010). En estos estudios se identifican las ventajas e inconvenientes de formar parte de un grupo de investigación consolidado o no consolidado. Entre las ventajas que citan los miembros de grupos consolidados se encuentran el intercambio de conocimientos; la amplitud de objetivos y líneas; la mejor organización, gestión, coordinación y/o planificación del grupo; la mayor productividad, rendimiento y competitividad; así como la mayor facilidad para obtener recursos humanos y económicos. Por su parte los inconvenientes señalan la menor libertad, autonomía e independencia del individuo, los problemas personales y el ambiente de trabajo y se centran asimismo en el tamaño y composición del equipo. Es decir, las ventajas se concentran en el plano científico, mientras que los inconvenientes apuntan más a una vertiente personal y social en la dinámica del grupo de investigación. En un trabajo previo del mismo autor centrado en la Geología (Rey-Rocha y otros, 2002), se mide la relación entre la productividad y el grado de consolidación de los grupos hallando que, más que el tamaño total del grupo, el factor diferenciador relacionado con la productividad es el número de personas dentro del mismo que alcanzan una posición estable y segura. Combinando técnicas cualitativas (encuestas) y bibliométricas en una muestra de geólogos españoles, los autores llegan a la conclusión que los grupos consolidados tienden a ser más numerosos, básicamente por contar con un mayor número de doctores, así como más productivos per cápita que los no consolidados. Formulan la hipótesis de que el mayor grado de consolidación que se produce en los grupos de más tamaño conduce a una mayor posibilidad de establecer colaboraciones con otros grupos lo que en última instancia favorecería una mayor productividad. En este sentido, parece indudable que la capacidad de atracción de recursos humanos y financiación, así como las posibilidades para personal en formación de trazar una carrera científica exitosa son mayores en un grupo consolidado. No hay que olvidar que uno de los factores esenciales en el otorgamiento de becas de formación de personal investigador es el currículo vitae del investigador principal y de su grupo de investigación. En la misma línea, Martín-Sempere y otros (2008) analizan la relación entre la consolidación de los equipos de investigación y la integración de los científicos en dichos grupos con diversos factores como la productividad, la colaboración o las patentes solicitadas. Los autores concluyen que los niveles más altos de integración en grupos consolidados se relacionan con una mayor productividad, así como con una participación más elevada en proyectos de investigación, y con un mayor número de tesis dirigidas. Sin embargo, cuando se trata de determinar el impacto de dichos grupos, son tanto en Biología-Biomedicina como en Química los no consolidados (grupos de menor tamaño) los que presentan mayores índices de impacto. Dicho de otra manera, los grupos más pequeños y menos productivos parecen rendir mejor en términos de visibilidad de la investigación que los grupos más grandes y más productivos (Rey-Rocha y otros, 2006; Rey-Rocha y otros, 2010).

Por su parte, también dentro del territorio español, se han realizado estudios sobre los grupos de investigación en la Comunidad Valenciana (GarcíaAracil y otros, 2006) analizando los factores que influyen en la concesión de la mención de excelencia, así como sobre la eficiencia de los equipos andaluces en el área de Tecnologías de la Información y Comunicación, usando técnicas de análisis envolvente de datos (Pino-Mejías y otros, 2010).

Así pues, la escasez de análisis que toman como caso de estudio los grupos de investigación españoles, unido a las evidencias contradictorias en lo 
que respecta a la relación entre rendimiento científico y tamaño del grupo de investigación, y la constatación de posibles diferencias entre disciplinas son las principales razones que justifican este análisis.

\section{OBJETIVOS}

El objetivo principal de este estudio es determinar si existe relación entre el tamaño del grupo de investigación y el rendimiento académico de sus componentes tanto para los indicadores absolutos como para los indicadores relativizados. Del mismo modo, dado los diferentes patrones de comportamiento bibliométrico que operan en las diferentes áreas del conocimiento (González-Albo y otros, 2012), se quiere calibrar si esta relación es generalizada o si es un hecho que se detecta únicamente en alguna de ellas. Esta información es relevante para la toma de decisiones dentro de las instituciones de educación superior, especialmente a la luz de la creciente importancia de los rankings universitarios que cada vez determinan en mayor grado la política científica de los centros de investigación.

Para responder a estas preguntas, se ha tomado como caso de estudio la Universidad de Murcia, una institución de carácter multidisciplinar (excluyendo las enseñanzas politécnicas) y de rendimiento medio, según los diferentes rankings de producción e impacto investigador (Torres-Salinas y otros, 2011; Buela-Casal y otros, 2011), que bien puede ser representativa de una importante parte de las universidades españolas.

\section{MATERIAL Y MÉTODOS}

Se ha realizado un estudio seccional descriptivo retrospectivo del rendimiento bibliométrico de los grupos de investigación de la Universidad de Murcia activos al menos durante un año para el periodo 2005-2009, excluyendo los pertenecientes al área de Humanidades $(n=258)$. Éstos se descartaron debido a la escasa representatividad de la producción recogida en la base de datos usada como fuente del estudio, así como a la inexistencia de factores de impacto calculados para las revistas de Humanidades y de indicadores de citas para el área en su conjunto. Se ha realizado un abordaje del grupo de investigación input-based (Cohen, 1991), bajo el que se registra la actividad científica de todos los miembros explícitamente declarados de una estructura administrativa con funciones investigadoras. En este caso es imprescindible contar con un censo previo de grupos de investigación y de sus integrantes. Esta perspectiva determinada por cuestiones administrativas se acerca más a los objetivos de la política científica, ya que a menudo es este nivel de agregación el que se considera a efectos de financiación de la investigación en estructuras universitarias o regionales. Para dicha población de grupos de investigación se calcularon dos indicadores de producción, dos de visibilidad y dos de impacto. Estos seis indicadores se pueden dividir entre indicadores absolutos, es decir, aquéllos que no están relativizados y por tanto, están influidos por el tamaño del grupo, e indicadores relativos, que en principio no están afectados por el tamaño de éste.

La información científica para realizar los cálculos bibliométricos se extrajo de las bases de datos ISI Web of Science, considerada la fuente más representativa para realizar estudios bibliométricos, por su selectividad y su carácter multidisciplinar, si bien presenta varias limitaciones, como la diferente cobertura según áreas temáticas (Moed, 2005), el sesgo anglosajón (van Leeuwen, 2001) o la falta de normalización en los nombres de los autores (Ruiz-Pérez y otros, 2002). Así, se descargaron todos los registros para el periodo 2005-2009 mediante la ecuación de búsqueda [(Murcia or Cartagena) and Spain], seleccionándose para el estudio únicamente las tipologías documentales artículo, revisión, carta y nota, consideradas tradicionalmente como ítems citables, e identificadas como las más relevantes en las evaluaciones bibliométricas (Schubert y otros, 1989). Dicha producción se revisó y filtró, descartándose los trabajos no vinculados a las personas censadas en los grupos de investigación de la Universidad de Murcia.

Hay que señalar que se realizó un acercamiento bottom-up de manera que la producción y actividad se asignó a cada investigador de forma individual. Esto se realizó incluso si el investigador no consignaba la afiliación institucional de la Universidad de Murcia en su firma en el artículo, hecho muy común sobre todo en investigadores con doble afiliación hospitalaria y universitaria. Seguidamente se descargó la información de las revistas agrupadas en los Journal Citation Reports (JCR) calculando asimismo el cuartil de cada revista para cada año y categoría donde ésta estuviera indizada. Igualmente se consignaron las citas de los artículos objeto de estudio; dicha búsqueda se realizó en febrero de 2011 para toda la producción, de manera que la ventana de citación es variable en función del año de publicación, y disponiendo por tanto los trabajos de más antigüedad de más tiempo para ser citados.

Los datos acerca de los grupos de investigación y la composición de cada uno de ellos fueron descargados desde la aplicación CURIE de la Universidad de Murcia, realizando actualizaciones anuales para dar cabida a los nuevos grupos, así como reseñando las altas y bajas de personal en los mismos. Toda esta información se integró en una única base de datos en formato MS Access 2003.

Los indicadores utilizados fueron:

PROD: Producción de los miembros del grupo de investigación, usando el método de recuento total.

PROD PER CAPITA: Producción per cápita del grupo de investigación. Se obtiene dividiendo el 
total de producción por el promedio de miembros anual del grupo.

Q1: Número de trabajos en revistas del primer cuartil, según la ordenación por factor de impacto para cada una de las categorías y año.

PORC_Q1: Porcentaje de trabajos en revistas del primer cuartil.

CITAS: Número total de citas recibidas por todos los trabajos de cada grupo de investigación.

CITAS_NORM: Valor de citas normalizado para cada grupo de investigación. El procedimiento de normalización de citas se realizó tomando como base la información de los Essential Science Indicators (ESI) a fecha enero de 2011, cubriendo el periodo temporal 2000-2010. A cada uno de los grupos de investigación se le clasificó en una de las 22 áreas definidas por ESI. Posteriormente para cada trabajo se dividió el número de citas reales recibidas por el valor correspondiente a su área científica y año. De este modo un valor de $1 \mathrm{co-}$ rrespondería al promedio mundial para su área y año. Finalmente se calculó el valor de citas normalizado para cada grupo de investigación.

El cálculo de los indicadores se realizó con MS Access 2003 mientras que para el análisis estadístico se ha usado el paquete informático SPSS en su versión 20.0. Además del cálculo de estadísticas descriptivas, el análisis estadístico se complementó con la prueba de Kruskal-Wallis. Dicho test es un estadístico no paramétrico, usado cuando las desviaciones estadísticas de las diferentes muestras son heterogéneas, y permite verificar si existen diferencias estadísticamente significativas entre ellas.

Los datos se relativizaron por el número de catedráticos de universidad, titulares de universidades, profesores contratados doctores y profesores ayudantes doctores del grupo. Estas escalas han sido las únicas contempladas ya que son las únicas donde es requisito indispensable ser doctor, y por tanto, tener encomendadas labores investigadoras. Así pues, siguiendo las directrices de von Tunzelmann y otros (2003), que afirman la dificultad de medir adecuadamente la labor del personal sin dedicación investigadora, se descartó usar el número total de componentes del grupo como medida del mismo, dada la naturaleza dinámica del propio grupo de investigación (Johnston, 1994) con numeroso personal transitorio (becarios, técnicos) unidos temporalmente al grupo de investigación y que pueden distorsionar los resultados, especialmente en aquéllos de mayor tamaño. En el caso que nos ocupa, además el hecho de que la fuente de datos incluya a los colaboradores de cada grupo (miembros de otras instituciones) añade mayor dificultad a la hora de computar su producción. De este modo, el tamaño $(T)$ medio del grupo se calcula realizando el promedio anual de dichos miembros doctores.
Por su parte, los grupos de investigación se clasificaron en cinco áreas de investigación. Se trata de una clasificación ad-hoc realizada a partir de las áreas de investigación existentes en la universidad objeto de estudio. Las cinco áreas son Física-Química (área 1), Recursos Naturales (incluyendo Biología Vegetal y Animal, Bioquímica y Veterinaria, área 2), Medicina Clínica y Salud (área 3), Matemáticas-Tecnologías de la Información y Comunicación (TIC) (área 4) y Ciencias Sociales (incluye Economía, Psicología, Educación y Otras Ciencias Sociales, área 5).

Para la categorización de los grupos en función del tamaño, se tomó la población de grupos de investigación para cada una de las áreas y se calcularon los indicadores de tercer cuartil estadístico, mediana y primer cuartil estadístico del promedio anual de miembros doctores, al objeto de clasificar los grupos de investigación en cuatro categorías aproximadamente iguales para cada área. De este modo, los grupos clasificados como A son los grupos de mayor tamaño, los grupos B presentan un tamaño medio-alto, los grupos $C$ un tamaño medio-bajo y los grupos $\mathrm{D}$ son los grupos más pequeños de la población estudiada.

\section{RESULTADOS}

La tabla I muestra la distribución de los 258 grupos de investigación según áreas científicas, así como los estadísticos relativos al tamaño de cada grupo de investigación. El área con mayor número de grupos es Ciencias Sociales, con un tercio de los grupos analizados, seguida de Recursos Naturales, con el $24,8 \%$ de los casos estudiados. El área más pequeña, con 19 grupos es el que abarca a las disciplinas de Matemáticas, y Tecnologías de la Información y Comunicación (TIC). El número medio de doctores por grupo se sitúa en 3,3 personas para el conjunto de los grupos de investigación, marcando Matemáticas-TIC $(4,3)$ y Medicina Clínica-Salud $(2,7)$ los valores promedio máximos y mínimos respectivamente. Hay que resaltar asimismo que en cuatro ocasiones (una en Recursos Naturales, dos en Medicina Clínica-Salud y una en Ciencias Sociales), se detectaron grupos sin ningún miembro en las escalas de catedrático, profesor titular, contratado doctor o ayudante doctor. Estos grupos se consideraron en el análisis de los diferentes indicadores.

En función del tamaño de los grupos de investigación, éstos se han agrupado en cuatro categorías, siendo los grupos A los de mayor tamaño y los $D$, los que menor número de componentes presentan. La tabla II muestra el número de grupos y el porcentaje que representan por áreas y categorías, así como el tamaño necesario para formar parte de cada una de las agrupaciones. La categoría $\mathrm{D}$ presenta el mayor número de grupos, 72, que significan el $27,9 \%$ del total analizado mientras que los grupos de mayor tamaño constituyen la categoría más pequeña, representando el $22,9 \%$ de casos 
estudiados. En cuanto al número de miembros de los grupos, existen diferencias significativas entre algunas de las categorías, marcando MatemáticasTIC $(5,7)$ y Física-Química (5) los valores más elevados para ser categorizados como A, y Ciencias Sociales (4) y Medicina-Clínica-Salud (3) los umbrales más bajos. A efectos prácticos, un grupo con un promedio de 4,2 miembros doctores $(T=4,2)$ estaría categorizado como B (tamaño medio-alto) en Física-Química, Recursos Naturales y Matemáticas-TIC mientras que en Medicina Clínica-Salud y en Ciencias Sociales, se clasificaría como grupo A (tamaño alto).

En la tabla III se señalan los indicadores absolutos de producción, visibilidad e impacto para cada una de las categorías definidas. En lo que respecta al indicador de producción total del grupo de investigación, la media se sitúa en 14,1 artículos por grupo, siendo muy inferior este promedio en Ciencias Sociales respecto al resto de áreas científicas. En todos los casos, salvo en Medicina ClínicaSalud (área 3), los grupos de mayor tamaño (A) presentan indicadores significativamente más elevados que el resto de categorías En el caso de esta área son los grupos clasificados como B (tamaño medio-alto) los que presentan una ratio superior, aunque con una desviación típica muy elevada. El segundo indicador mostrado, el número de trabajos en revistas del primer cuartil por categorías JCR también señala la asociación entre tamaño de grupo y rendimiento, tanto a nivel global como por áreas científicas. La única salvedad se localiza en el área 2 (Recursos Naturales), donde los grupos de tamaño medio-bajo (C) presentan una ratio ligeramente superior a la de los grupos de tamaño medio-grande (B). Por su parte, el número total de citas cosechado por grupo de investigación señala la misma tendencia. Los grupos A presentan de media casi 160 citas, frente a las algo más de 100 de los grupos B, las 72,9 menciones de los grupos de tamaño medio-bajo (C), y las 34,6 citas de los grupos pequeños (D). Como excepción, hay que señalar de nuevo en el área de Recursos Naturales (área 2) la mejor ratio de los grupos C sobre los B, y en el caso de Medicina Clínica-Salud el valor más elevado que presentan los grupos B sobre los A, si bien como se ha comentado previamente con una alta desviación estadística sobre los valores medios de la categoría.

Tabla I. Estadísticas descriptivas del tamaño del grupo de investigación

\begin{tabular}{ccccccc}
\hline & \multicolumn{7}{c}{ ÁREA } \\
\hline INDICADOR & $\mathbf{1}$ & $\mathbf{2}$ & $\mathbf{3}$ & $\mathbf{4}$ & $\mathbf{5}$ & TOTAL \\
\hline $\mathrm{N}$ & 35 & 64 & 55 & 19 & 85 & 258 \\
\hline$\%$ & 13,6 & 24,8 & 21,3 & 7,4 & 32,9 & 100,0 \\
\hline MEDIA \pm DT & $3,7 \pm 2,2$ & $3,5 \pm 1,8$ & $2,7 \pm 2,1$ & $4,3 \pm 2,5$ & $3,3 \pm 2,2$ & $3,3 \pm 2,1$ \\
\hline RANGO & $1,0-10,4$ & $0,0-9,8$ & $0,0-10,8$ & $1,0-9,4$ & $0,0-10,0$ & $0,0-10,8$ \\
\hline
\end{tabular}

$\mathrm{N}=$ Número de grupos; \%=Porcentaje de grupos; Media \pm DT=Media y desviación típica del tamaño del grupo de investigación; Áreas: 1.Física-Química; 2. Recursos Naturales; 3. Medicina Clínica; 4. Matemáticas-TIC; 5. Ciencias Sociales.

Tabla II. Grupos de investigación según tamaño (número de doctores) y áreas científicas

\begin{tabular}{|c|c|c|c|c|c|c|c|}
\hline \multirow[b]{2}{*}{ CATEGORÍA } & \multirow[b]{2}{*}{ INDICADOR } & \multicolumn{6}{|c|}{ ÁREA } \\
\hline & & 1 & 2 & 3 & 4 & 5 & TOTAL \\
\hline \multirow{3}{*}{ A } & $\mathrm{N}$ & 7 & 15 & 13 & 5 & 19 & 59 \\
\hline & $\%$ & 20,0 & 23,4 & 23,6 & 26,3 & 22,4 & 22,9 \\
\hline & $\mathrm{T}$ & {$[10,4-5,0)$} & {$[9,8-4,8)$} & {$[10,8-3,0)$} & {$[9,4-5,7)$} & {$[10,0-4,0)$} & \\
\hline \multirow{3}{*}{ B } & $\mathrm{N}$ & 9 & 17 & 10 & 4 & 20 & 60 \\
\hline & $\%$ & 25,7 & 26,6 & 18,2 & 21,1 & 23,5 & 23,3 \\
\hline & $\mathrm{T}$ & {$[5,0-3,0)$} & {$[4,8-3,1)$} & {$[3,0-2,0)$} & {$[5,7-3,8)$} & {$[4,0-3,0)$} & \\
\hline \multirow{3}{*}{ C } & $\mathrm{N}$ & 8 & 14 & 18 & 5 & 22 & 67 \\
\hline & $\%$ & 22,9 & 21,9 & 32,7 & 26,3 & 25,9 & 26,0 \\
\hline & $\mathrm{T}$ & {$[3,0-2,0)$} & {$[3,1-2,0)$} & {$[2,0-1,2)$} & {$[3,8-2,3)$} & {$[3,0-1,8)$} & \\
\hline \multirow{3}{*}{ D } & $\mathrm{N}$ & 11 & 18 & 14 & 5 & 24 & 72 \\
\hline & $\%$ & 31,4 & 28,1 & 25,5 & 26,3 & 28,2 & 27,9 \\
\hline & $\mathrm{T}$ & {$[2,0-1,0]$} & {$[2,0-0,0]$} & {$[1,2-0,0]$} & {$[2,3-1,0]$} & {$[1,8-0,0]$} & \\
\hline TOTAL & $\mathrm{N}$ & 35 & 64 & 55 & 19 & 85 & 258 \\
\hline
\end{tabular}

Categoría: A. Grupos de tamaño alto; B. Grupos de tamaño medio-alto; C. Grupos de tamaño medio-bajo; D. Grupos de tamaño bajo; N=Número de grupos; \%=Porcentaje de grupos; $\mathrm{T}=$ Intervalo del tamaño del grupo de investigación; Áreas: 1. FísicaQuímica; 2. Recursos Naturales; 3. Medicina Clínica; 4. Matemáticas-TIC; 5. Ciencias Sociales. 
Por su parte, los indicadores relativos de producción, visibilidad e impacto señalan escasas diferencias entre los grupos de distinto tamaño (tabla IV). Así, en el indicador de producción per cápita, son, a nivel general los grupos de tamaño medio-alto (B) los que presentan el indicador más elevado $(5,0)$. Si se ahonda en cada una de las áreas científicas, los grupos B presentan el mejor dato en todas las áreas, salvo en Recursos Naturales (área 2), donde dominan los grupos de tamaño medio-bajo (C). Por su parte, en las áreas de Matemáticas-TIC (área 4) y Ciencias Sociales (área 5) esta ratio es idéntica a la de los grupos de mayor tamaño (A). En lo que respecta al indicador de visibilidad, porcentaje de trabajos en revistas del primer cuartil, son los grupos de mayor tamaño (A) los que señalan el mejor dato, con un $36,6 \%$ de sus trabajos en dichas publicaciones de prestigio. El segundo mejor dato promedio lo presentan los grupos más pequeños, con un $29,4 \%$ de artículos en revistas de alta visibilidad, si bien con un dato que apenas supera ligeramente a las otras dos categorías de grupos. En el análisis por áreas, se detectan patrones muy diferenciados. Por un lado, tanto en Física-Química (área 1) como en Ciencias Sociales (área 5), el mayor tamaño del grupo se asocia con una mayor ratio de publicación en revistas de alta visibilidad, mientras que en Matemáticas-TIC (área 4), esta relación es inversa, es decir, son los grupos más pequeños los que muestran la mayor eficiencia en publicación en revistas de prestigio. En las dos restantes áreas no se detectan tendencias claras. Finalmente, el indicador de citas normalizadas señala a los grupos de tamaño alto (A) y medio-bajo (C) como los únicos que alcanzan los promedios mundiales de citación. Sin embargo, el valor que alcanzan los grupos de tamaño medio-bajo (C) está muy determinado por los valores extremos de Física-Química $(3,3)$, y que se refleja en una elevadísima desviación típica sobre los valores medios. Excluyendo el área de Ciencias Sociales, que queda muy alejado de los promedios mundiales de citación, en el resto de áreas, los grupos de mayor tamaño (A) presentan valores medios siempre por encima de la ratio mundial, algo que no ocurre en las restantes categorías.

Tabla III. Media y desviación típica de los indicadores absolutos de producción, visibilidad e impacto según tamaño y área de los grupos de investigación

\begin{tabular}{|c|c|c|c|c|c|c|c|}
\hline \multirow[b]{2}{*}{ VARIABLE } & \multirow[b]{2}{*}{ CATEGORÍA } & \multicolumn{6}{|c|}{ ÁREA } \\
\hline & & 1 & 2 & 3 & 4 & 5 & TOTAL \\
\hline \multirow{5}{*}{ PROD } & A & $32,6 \pm 16,1$ & $33,5 \pm 18,8$ & $30,1 \pm 23,7$ & $37,4 \pm 17,0$ & $8,7 \pm 11,9$ & $25,0 \pm 20,6$ \\
\hline & B & $29,3 \pm 15,9$ & $18,6 \pm 13,7$ & $32,9 \pm 50,4$ & $21,8 \pm 8,5$ & $5,0 \pm 7,4$ & $18,3 \pm 24,7$ \\
\hline & $C$ & $14,1 \pm 7,1$ & $17,6 \pm 8,8$ & $11,5 \pm 20,9$ & $13,2 \pm 8,4$ & $1,8 \pm 2,5$ & $10,0 \pm 13,3$ \\
\hline & $D$ & $9,7 \pm 10,0$ & $7,8 \pm 7,2$ & $6,6 \pm 5,8$ & $5,8 \pm 5,1$ & $0,7 \pm 1,1$ & $5,3 \pm 6,8$ \\
\hline & TOTAL & $20,3 \pm 15,6$ & $18,8 \pm 15,6$ & $18,5 \pm 28,6$ & $19,4 \pm 15,8$ & $3,8 \pm 7,4$ & $14,1 \pm 18,7$ \\
\hline \multirow{5}{*}{ Q1 } & $A$ & $24,0 \pm 14,9$ & $16,3 \pm 14,9$ & $14,9 \pm 15,0$ & $7,6 \pm 6,7$ & $1,5 \pm 2,7$ & $11,4 \pm 13,8$ \\
\hline & B & $19,6 \pm 11,2$ & $9,0 \pm 6,5$ & $7,0 \pm 10,8$ & $6,8 \pm 4,5$ & $0,5 \pm 0,8$ & $7,3 \pm 9,3$ \\
\hline & C & $8,0 \pm 5,2$ & $9,1 \pm 4,6$ & $3,5 \pm 7,2$ & $5,2 \pm 3,4$ & $0,1 \pm 0,4$ & $4,2 \pm 5,8$ \\
\hline & D & $4,9 \pm 4,8$ & $3,8 \pm 5,3$ & $2,1 \pm 2,2$ & $2,4 \pm 2,7$ & $0,0 \pm 0,0$ & $2,3 \pm 3,8$ \\
\hline & TOTAL & $13,2 \pm 12,0$ & $9,3 \pm 9,6$ & $6,5 \pm 10,6$ & $5,4 \pm 4,7$ & $0,5 \pm 1,4$ & $6,0 \pm 9,3$ \\
\hline \multirow{5}{*}{ CITAS } & $A$ & $330,3 \pm 293,3$ & $224,3 \pm 209,8$ & $182,7 \pm 205,7$ & $124,2 \pm 125,2$ & $34,9 \pm 74,6$ & $158,2 \pm 202,5$ \\
\hline & B & $221,6 \pm 174,9$ & $106,7 \pm 109,3$ & $184,9 \pm 309,9$ & $47,5 \pm 20,0$ & $14,8 \pm 33,3$ & $102,4 \pm 169,0$ \\
\hline & $\mathrm{C}$ & $213,4 \pm 316,8$ & $112,8 \pm 89,4$ & $71,8 \pm 148,8$ & $44,4 \pm 38,3$ & $3,8 \pm 8,6$ & $72,9 \pm 149,7$ \\
\hline & D & $62,2 \pm 102,0$ & $64,9 \pm 78,1$ & $38,1 \pm 38,3$ & $19,0 \pm 22,6$ & $0,5 \pm 1,2$ & $34,6 \pm 63,2$ \\
\hline & TOTAL & $191,3 \pm 236,3$ & $123,8 \pm 140,1$ & $110,0 \pm 192,0$ & $59,4 \pm 75,5$ & $12,4 \pm 40,5$ & $88,6 \pm 157,2$ \\
\hline
\end{tabular}

Categoría: A. Grupos de tamaño alto; B. Grupos de tamaño medio-alto; C. Grupos de tamaño medio-bajo; d. Grupos de tamaño bajo; Áreas: 1.Física-Química; 2. Recursos Naturales; 3. Medicina Clínica; 4. Matemáticas-TIC; 5 . Ciencias Sociales. 
La prueba de Kruskal-Wallis permite contrastar si existen diferencias estadísticamente significativas entre k muestras independientes. En este caso las muestras son los indicadores alcanzados por cada una de las cuatro tipologías de grupo definidas en función de su tamaño (tabla V). Dicho test señala cómo existen diferencias estadísticamente significativas a nivel global para las tres variables absolutas calculadas $(p<0,05)$ así como para la mayor parte de áreas. La excepción se encuentra en el área Matemáticas-TIC (área 4), donde las diferencias no son significativas en el caso de la publicación en revistas del primer cuartil, y lo son en cuanto al número total de citas con $p<0,1$. En cuanto a los valores relativos encontramos diferen- cias significativas en cuanto al valor de citas normalizadas con un intervalo de confianza (CI) del $95 \%$, mientras que estas diferencias también son significativas para el porcentaje de trabajos en revistas del primer cuartil para $\mathrm{CI}=90 \%$. Si se ahonda en las diferentes áreas, en la mayoría no se producen diferencias significativas entre grupos de diferente tamaño. Las salvedades se producen en cuanto al indicador de porcentaje de trabajos en revistas del primer cuartil en el área de Medicina Clínica y Salud, y en el área de Ciencias Sociales, donde se dan diferencias estadísticamente significativas en los tres indicadores relativos con un intervalo de confianza del $95 \%$.

Tabla IV. Media y desviación típica de los indicadores relativos de producción, visibilidad e impacto según tamaño y área de los grupos de investigación

\begin{tabular}{|c|c|c|c|c|c|c|c|}
\hline \multirow[b]{2}{*}{ VARIABLE } & \multirow[b]{2}{*}{ CATEGORÍA } & \multicolumn{6}{|c|}{ ÁREA } \\
\hline & & 1 & 2 & 3 & 4 & 5 & TOTAL \\
\hline \multirow{5}{*}{ PROD PER CAPITA } & A & $4,7 \pm 2,7$ & $5,8 \pm 3,6$ & $6,0 \pm 5,0$ & $4,8 \pm 2,3$ & $1,3 \pm 1,7$ & $4,2 \pm 3,8$ \\
\hline & B & $6,6 \pm 3,2$ & $4,9 \pm 4,0$ & $11,0 \pm 16,8$ & $4,8 \pm 1,5$ & $1,3 \pm 1,9$ & $5,0 \pm 7,8$ \\
\hline & C & $5,0 \pm 2,4$ & $6,2 \pm 3,2$ & $6,0 \pm 10,5$ & $4,0 \pm 2,3$ & $0,7 \pm 0,9$ & $4,0 \pm 6,1$ \\
\hline & D & $6,3 \pm 5,2$ & $5,2 \pm 4,9$ & $6,6 \pm 5,8$ & $4,0 \pm 3,8$ & $0,5 \pm 1,0$ & $4,0 \pm 4,8$ \\
\hline & TOTAL & $5,8 \pm 3,6$ & $5,5 \pm 4,0$ & $7,1 \pm 10,0$ & $4,4 \pm 2,5$ & $0,9 \pm 1,4$ & $4,3 \pm 5,8$ \\
\hline \multirow{5}{*}{ PORC_Q1 } & A & $70,5 \pm 15,6$ & $44,3 \pm 20,7$ & $42,9 \pm 17,3$ & $24,2 \pm 20,7$ & $17,1 \pm 30,6$ & $36,6 \pm 28,5$ \\
\hline & B & $60,0 \pm 24,3$ & $45,2 \pm 21,6$ & $15,6 \pm 17,0$ & $34,0 \pm 23,0$ & $4,8 \pm 9,9$ & $28,3 \pm 27,6$ \\
\hline & C & $54,5 \pm 21,4$ & $51,6 \pm 15,0$ & $27,9 \pm 34,4$ & $37,3 \pm 28,2$ & $3,8 \pm 11,5$ & $28,8 \pm 29,9$ \\
\hline & D & $51,8 \pm 26,9$ & $34,5 \pm 26,8$ & $24,4 \pm 21,7$ & $55,2 \pm 45,1$ & $0,0 \pm 0,0$ & $25,1 \pm 29,8$ \\
\hline & TOTAL & $58,3 \pm 23,2$ & $43,4 \pm 22,2$ & $28,3 \pm 26,2$ & $37,9 \pm 30,9$ & $5,9 \pm 17,2$ & $29,4 \pm 29,1$ \\
\hline \multirow{5}{*}{ CITAS_NORM } & A & $1,4 \pm 0,6$ & $1,1 \pm 0,6$ & $1,1 \pm 0,7$ & $1,5 \pm 1,1$ & $0,5 \pm 0,6$ & $1,0 \pm 0,8$ \\
\hline & B & $1,0 \pm 0,6$ & $0,8 \pm 0,3$ & $0,5 \pm 0,5$ & $1,2 \pm 0,6$ & $0,5 \pm 0,6$ & $0,7 \pm 0,5$ \\
\hline & C & $3,3 \pm 4,7$ & $1,1 \pm 0,6$ & $0,8 \pm 0,8$ & $1,3 \pm 0,7$ & $0,2 \pm 0,3$ & $1,0 \pm 1,9$ \\
\hline & D & $1,1 \pm 1,1$ & $0,9 \pm 0,8$ & $0,5 \pm 0,4$ & $2,7 \pm 2,8$ & $0,1 \pm 0,3$ & $0,7 \pm 1,1$ \\
\hline & TOTAL & $1,6 \pm 2,4$ & $1,0 \pm 0,6$ & $0,7 \pm 0,7$ & $1,7 \pm 1,6$ & $0,3 \pm 0,5$ & $0,8 \pm 1,2$ \\
\hline
\end{tabular}

Categoría: A. Grupos de tamaño alto; B. Grupos de tamaño medio-alto; C. Grupos de tamaño medio-bajo; d. Grupos de tamaño bajo; Áreas: 1.Física-Química; 2. Recursos Naturales; 3. Medicina Clínica; 4. Matemáticas-TIC; 5. Ciencias Sociales.

Tabla V. Significación estadística de la prueba de Kruskal-Wallis para los indicadores de producción, visibilidad e impacto por áreas científicas

\begin{tabular}{cccccccc}
\hline & \multicolumn{7}{c}{ ÁREA } \\
\hline INDICADORES & VARIABLE & $\mathbf{1}$ & $\mathbf{2}$ & $\mathbf{3}$ & $\mathbf{4}$ & $\mathbf{5}$ & TOTAL \\
\hline \multirow{3}{*}{ ABSOLUTOS } & PROD & $0,003 *$ & $0,000 *$ & $0,005 *$ & $0,008^{*}$ & $0,000 *$ & $0,000 *$ \\
\cline { 2 - 8 } & Q1 & $0,002 *$ & $0,001 *$ & $0,003 *$ & 0,350 & $0,001 *$ & $0,000 *$ \\
\cline { 2 - 8 } & CITAS & $0,011 *$ & $0,011 *$ & $0,025 *$ & $0,084 * *$ & $0,006 *$ & $0,000 *$ \\
\hline \multirow{3}{*}{ RELATIVOS } & PROD PER CAPITA & 0,664 & 0,488 & 0,561 & 0,900 & $0,049 *$ & 0,362 \\
\cline { 2 - 8 } & PORC_Q1 & 0,342 & 0,208 & $0,025 *$ & 0,665 & $0,001 *$ & $0,059 * *$ \\
\cline { 2 - 8 } & CITAS_NORM & 0,664 & 0,549 & 0,112 & 0,861 & $0,009 *$ & $0,012 *$ \\
\hline
\end{tabular}

*Diferencias significativas $\mathrm{p}<0,05 ; * *$ Diferencias significativas $\mathrm{p}<0,1 ;$ Áreas: 1.Física-Química; 2. Recursos Naturales; 3. Medicina Clínica; 4. Matemáticas-TIC; 5. Ciencias Sociales. 


\section{DISCUSIÓN Y CONCLUSIONES}

El presente estudio se ha focalizado en un aspecto muy determinado de la actividad investigadora a nivel micro, como es la relación entre el número de miembros de los grupos de investigación y su relación con los parámetros bibliométricos. Existen otros factores que pueden afectar al rendimiento del grupo de investigación y que no se han estudiado en este trabajo tales como la escala profesional del investigador principal del grupo, el sexo, la edad, las relaciones que se establecen entre los componentes del grupo de investigación, o el acceso a equipos o material técnico especializado, entre otros. Asimismo el análisis se ha centrado en una única universidad, por lo que hay que ser precavido a la hora de hacer extensibles estos resultados de forma global a los grupos universitarios españoles, y tomar estos resultados solo a modo indicativo. Del mismo modo, hay que señalar otras limitaciones como la inclusión únicamente de artículos internacionales, lo que puede afectar sobre todo a grupos de Ciencias Sociales que pueden tener en la publicación en revistas nacionales o en monografías su vía de expresión principal. En definitiva esta aportación debe entenderse como una propuesta metodológica para aproximarse a algunas de las variables principales, si bien no las únicas, que afectan al estudio de los grupos de investigación. Igualmente, a la hora de interpretar estos datos hay que tener muy en cuenta que dentro de cualquier universidad española conviven grupos con un alto rendimiento con otros grupos de rendimiento medio o bajo, como se desprende de las elevadas desviaciones de la media en algunas áreas halladas en este estudio. Para el sistema español se podría aplicar la máxima que se expone para Italia (Abramo y otros, 2011) y es que existen mayores diferencias entre los grupos dentro de la propia universidad que entre las distintas universidades, por lo que una de las primeras políticas a aplicar por cualquier institución sería la de identificar y monitorizar el rendimiento de dichos grupos de investigación a fin de establecer incentivos adecuados de cara a su mejora.

Los resultados hallados señalan para el caso de los indicadores absolutos la dependencia del tamaño del grupo de investigación. Esto no es una sorpresa ya que cuanto mayor es el número de investigadores del equipo de investigación, mayor posibilidad de producir trabajos, de publicarlos en revistas de alta visibilidad, y de recibir citas por ellos. Así, los grupos de mayor tamaño presentan unos indicadores absolutos que multiplican por un factor de entre 4,6 y 5 (en función del indicador considerado) el valor de los grupos de menor tamaño. Estos resultados son estadísticamente significativos salvo en el caso de Matemáticas-TIC donde el tamaño no parece ejercer un efecto en líneas generales sobre dichos indicadores.

En cuanto a los resultados en los indicadores relativos, el análisis señala que a nivel general el ta- maño del grupo no afecta la productividad de éste, mientras que la eficiencia en la publicación en revistas del primer cuartil, y las citas recibidas ajustadas por trabajo y área sí se ven influidas por el tamaño del equipo de investigación. Estos resultados sin embargo están plenamente condicionados por el comportamiento de las Ciencias Sociales, que representan un tercio de los grupos de investigación estudiados, ya que a nivel de área, sólo en ésta, y en Medicina Clínica y Salud (porcentaje en revistas del primer cuartil), afecta el tamaño del grupo.

Un análisis más pormenorizado de los resultados en Ciencias Sociales señala que los indicadores y patrones que se producen en el área se alejan de lo hallado en los restantes campos científicos. En dicha área, la producción científica internacional es escasa, y también son muy bajos los indicadores de visibilidad e impacto. Por el contrario, existe un elevado número de grupos de investigación que no presentan producción internacional, y que por tanto también muestran indicadores de visibilidad e impacto iguales a cero. Dichos grupos, que tienden a concentrarse en la categoría $\mathrm{D}$, esto es, son de muy pequeño tamaño (presentan como máximo 1,8 investigadores por año), ejemplarizan la atomización de la investigación en Ciencias Sociales. Esta atomización parece afectar de forma negativa al área, ya que la productividad y visibilidad internacional de estos grupos es escasa o nula. El hecho de que sean los grupos de mayor tamaño los que muestran una mayor productividad y eficiencia en la publicación señala que son dichos equipos de investigación los que están adoptando en mayor medida un patrón de publicación internacional. Nuestros datos no permiten afirmar de manera fehaciente la causa de esta mayor eficiencia de los grupos de mayor tamaño, aunque podría relacionarse con el concepto de masa crítica necesaria para ser competitivos internacionalmente. Este factor puede jugar un papel más importante en los grupos de Ciencias Sociales dado el menor número de colaboraciones que se producen en el área respecto a las ciencias básicas. Igualmente, estos datos son significativos de cara a la realización de una política científica que en dicha área prime la agregación de investigadores dentro de un grupo de investigación, si bien existen riesgos de que se formen grupos "administrativos" o artificiales, carentes de factores sociales tales como integración o cohesión grupal, que juegan un papel relevante en el desempeño del grupo de investigación (ReyRocha y otros, 2008).

En el resto de áreas sin embargo hay escasa evidencia de que incentivar grupos de investigación de tamaño elevado redunde en una mejora de la productividad y eficiencia de sus miembros, por lo que se hace necesario buscar otros factores que expliquen el mejor desempeño de unos grupos de investigación sobre otros. No hay que obviar, sin embargo, que son los grupos de mayor tamaño los que generan el mayor output científico de una ins- 
titución (los grupos A producen el $40,7 \%$ de los artículos científicos en este estudio), por lo que una opción dentro de la política científica podría ser incidir en mejorar la eficiencia en términos de visibilidad e impacto de los grupos de investigación más prolíficos.

En cualquier caso, no hay que perder de vista que las recompensas en la ciencia española se basan no tanto en la eficiencia, sino principalmente en el cumplimiento o superación de unos umbrales mínimos de publicación, y que esta evaluación se realiza a nivel individual, no de grupo de investigación (Fernández-Esquinas y otros, 2006). Así ocurre con los sexenios de investigación otorgados por la Comisión Nacional Evaluadora de la Actividad Investigadora (CNEAI), en que se someten cinco trabajos a evaluación (Ruiz-Pérez y otros, 2010, Ministerio de Educación, 2011) o en la promoción académica en la universidad española que fija un número de publicaciones mínimo en función de la escala a la que aspira el candidato (ANECA, 2008). Sin embargo, superado estos umbrales, los investigadores disponen de escasos incentivos para aumentar su productividad y visibilidad. Así pues, desde un punto de vista individual a un investigador le podría compensar formar parte de un grupo de elevado tamaño sobre uno pequeño, ya que en éste dispondrá de más oportunidades para realizar artículos de investigación, publicarlos en revista de alta visibilidad y recibir recompensas académicas por ello.

Finalmente, es capital la realización de más estudios bibliométricos con enfoque en la política científica, tanto a nivel meso (Filippo y otros, 2012; Docampo y otros, 2012) como micro (Costas y Bordons, 2005; Costas y otros, 2010) que identifiquen los factores que influyen en el rendimiento de los agregados de investigación, a fin de que las instituciones puedan adoptar medidas que contribuyan a aumentar no sólo la productividad de la investigación en las universidades, sino especialmente su visibilidad e impacto internacional.

\section{BIBLIOGRAFÍA}

ANECA. (2008). Principios y Orientaciones para la Aplicación de los Criterios de Evaluación.

Abramo, G.; Cicero, T.; D'Angelo, C. A. (2011). The dangers of performance-based research funding in non-competitive higher education systems. Scientometrics, vol. 87 (3), 641-654.

Buela-Casal, G.; Bermúdez, M. P.; Sierra, J. C.; Quevedo-Blasco, R.; Castro, Á.; Guillén-Riquelme, A. (2011). Ranking de 2010 en producción y productividad en investigación de las universidades públicas españolas. Psicothema, vol. 23 (4), 527-536.

Cohen, J. E. (1991). Size, age and productivity of scientific and technical research groups. Scientometrics, vol. 20 (3), 395-416.

Costas, R.; Bordons, M. (2005). Bibliometric indicators at the micro-level: Some results in the area of natural resources at the Spanish CSIC. Research Evaluation, vol. 14 (2), 110-120.

Costas, R.; Van Leeuwen, T. N.; Bordons, M. (2010). A bibliometric classificatory approach for the study and assessment of research performance at the individual level: the effects of age on productivity and impact. Journal of the American Society for Information Science and Technology, vol. 61 (8), 1564-1581.

Docampo, D.; Herrera, F.; Luque-Martínez, T.; TorresSalinas, D. (2012). Efecto de la agregación de universidades españolas en el Ranking de Shanghai (ARWU): caso de las comunidades autónomas y los campus de excelencia. El Profesional de la información, vol. 21 (4), 428-432.

Dundar, H.; Lewis, D. R. (1998). Determinants of research productivity in higher education. Research in Higher Education, vol. 39 (6), 607-631.

Etzkowitz, H. (1992). Individual investigators and their research groups. Minerva, vol. 30 (1), 28-50.

Fernández-Esquinas, M.; Pérez-Yruela, M.; MerchánHernández, C. (2006). El Sistema de Incentivos y Recompensas en la Ciencia Pública Española. En J. Sebastián y E. Muñoz (editores) Radiografía de la investigación pública en España. Biblioteca Nueva, Madrid.

Filippo, D. de; Casani, F.; García-Zorita, C.; EfraínGarcía, P.; Sanz-Casado, E. (2012). Visibility in international rankings. Strategies for enhancing the competitiveness of Spanish universities. Scientometrics.

García-Aracil, A.; Gracia, A. G.; Pérez-Marín, M. (2006). Analysis of the evaluation process of the research performance: An empirical case. Scientometrics, vol. 67 (2), 213-230.

Granovetter, M. S. (1973). The strength of weak ties. American Journal of Sociology, vol. 78 (6), 13601380.

González-Albo, B.; Moreno, L.; Morillo, F.; Bordons, M. (2012). Indicadores bibliométricos para el análisis de la actividad de una institución multidisciplinar: el CSIC. Revista Española de Documentación Científica, 35 (1), 9-37.

Johnston, R. (1994). Effects of resource concentration on research performance. Higher Education, vol. 28 (1), 25-37.

Kenna, R.; Berche, B. (2011). Critical mass and the dependency of research quality on group size. Scientometrics, vol. 86 (2), 527-540.

Kenna, R.; Berche, B. (2012). Managing research quality: critical mass and optimal academic research group size. IMA Journal of Management Mathematics, vol. 23 (2), 195-207.

Lee, S.; Bozeman, B. (2005). The impact of research collaboration on scientific productivity. Social Studies of Science, vol. 35 (5), 673-702.

Martín-Sempere, M. J.; Garzón-García, B.; Rey-Rocha, J. (2008). Team consolidation, social integration and scientists' research performance: An empirical study in the Biology and Biomedicine field. Scientometrics, vol. 76 (3), 457-482. 
Ministerio de Educación (2011). Resolución de 30 de noviembre de 2011, de la Secretaría General de Universidades, por la que se fija el procedimiento $y$ plazo de presentación de solicitudes de evaluación de la actividad investigadora a la Comisión Nacional Evaluadora de la Actividad Investigadora.

Moed, H. F. (2005). Citation analysis in research evaIuation. Dordrecht; Springer.

Orduña-Malea, E. (2011). Propuesta de un modelo de análisis redinformétrico multinivel para el estudio sistémico de las universidades españolas. [Tesis doctoral] Universidad Politécnica de Valencia.

Pino-Mejías, J.; Solís-Cabrera, F.; Delgado-Fernández, M.; Barea-Barrera, R. (2010). Evaluación de la eficiencia de grupos de investigación mediante análisis envolvente de datos (DEA). El Profesional de la Información, vol. 19 (2), 160-167.

Rey-Rocha, J.; Martín-Sempere, M. J.; Garzon, B. (2002). Research productivity of scientists in consolidated vs. non-consolidated teams: The case of Spanish university geologists. Scientometrics, vol. 55 (1), 137-156.

Rey-Rocha, J.; Martin-Sempere, M. J.; Sebastián, J. (2008). Estructura y dinámica de los grupos de investigación. Arbor, vol. 184 (732), 743-757.

Rey-Rocha, J.; Garzón-García, B.; Martín-Sempere, M. J. (2006). Consolidación y cohesión de los equipos de investigación del CSIC y su influencia sobre la actividad investigadora y el rendimiento de sus componentes: Área de Biología y Biomedicina. Informe CSIC-153pp.

Rey-Rocha, J.; Garzón-García, B.; Martín-Sempere, M. J. (2010). Consolidación y cohesión de los grupos de investigación del CSIC y su influencia sobre la actividad investigadora y el rendimiento de sus componentes: Área de Ciencia y Tecnologías Químicas. Informe CSIC-109pp.

Ruiz-Pérez, R.; Delgado-López-Cózar, E.; JiménezContreras, E. (2002). Spanish personal name variations in national and international biomedical da- tabases: implications for information retrieval and bibliometric studies. Journal of the Medical Library Association, vol. 90 (4), 411-430.

Ruiz-Pérez, R.; Delgado-López-Cózar, E.; JiménezContreras, E. (2010). Principios y criterios utilizados en España por la Comisión Nacional Evaluadora de la Actividad Investigadora (CNEAI) para la valoración de las publicaciones científicas: 1989-2009. Psicothema, vol. 22 (4), 898-908.

Schubert, A.; Glänzel, W.; Braun, T. (1989). A comprehensive set of indicators on 2649 journals and 96 countries in all major science fields and subfields 1981-1985. Scientometrics, vol. 16 (1-6), 3-478.

Seglen, P.; Aksnes, D. W. (2000). Scientific productivity and group size: A bibliometric analysis of Norwegian microbiological research. Scientometrics, vol. 49 (1), 125-143.

Torres-Salinas, D.; García-Moreno-Torres, J.; Robinson-García, N.; Delgado-López-Cózar, E.; Herrera, F. (2011). Rankings ISI de las universidades españolas según campos y disciplinas científicas ( $2^{\mathrm{a}}$ ed. 2011). El profesional de la información, vol. 20 (6), 701-709.

van Leeuwen, T.; Moed, H.; Tijssen, R.; Visser, M. (2001). Language biases in the coverage of the Science Citation Index and its consequences for international comparisons of national research performance. Scientometrics, vol. 51 (1), 335-346.

van Raan, A. F. J. (2006). Performance-related differences of bibliometric statistical properties of research groups: Cumulative advantages and hierarchically layered networks. Journal of the American Society for Information Science and Technology, vol. 57 (14), 1919-1935.

von Tunzelmann, N.; Ranga, M.; Martin, B.; Geuna, A. (2003). The Effects of Size on Research Performance: A SPRU Review. 